\title{
Measurements and Modeling of Temporal Variations of the Indoor Radio Propagation Channel
}

\author{
Homayoun Hashemi, Senior Member, IEEE, Michael McGuire, Thomas Vlasschaert, \\ David Tholl, Student Member, IEEE
}

\begin{abstract}
This paper reports the results of extensive measurements and analysis of the indoor radio propagation channel's temporal variations. The empirical data base consists of 192 one-min recordings of $\mathrm{CW}$ envelope fading waveforms with both antennas stationary. Measurements were carried out in an office environment at $1100 \mathrm{MHz}$ with four transmitter-receiver antenna separations of $5,10,20$, and $30 \mathrm{~m}$. Effects of controlled degrees of motion with $0,1,2,3$, or 4 individuals walking around the high antenna only, around the low antenna only, and around both antennas were investigated. The reported results include amplitude fading distributions, correlation properties, level crossing rates, duration of fades statistics, and spectrum widths of the frequency domain data. The results can be used in fixed wireless computer communication applications, and in supplementing the available spatial variation models of the indoor radio propagation channel.
\end{abstract}

\section{INTRODUCTION}

$\mathrm{D}$ ETAILED knowledge of radio propagation inside buildings is essential in the successful design of future PCS systems. Measurements and modeling of indoor radio propagation channels have been performed and reported by different investigators [1]-[8]. (Reference [1] is a comprehensive tutorial-survey coverage of the subject.) These reports are mainly based on characterization of the channel's impulse response. Detailed study of indoor channel characteristics when the mobile antenna changes position has been reported in [1]-[3] on the basis of 12000 impulse response profiles collected in two office environments.

When both antennas (fixed or "base" and mobile or “portable") are stationary, motion of people and equipment around the antennas result in multipath disturbances and fading effects [1]. This temporal fading phenomenon has been studied in [4]-[8]. CW measurements around 1 $\mathrm{GHz}$ in five factory environments [4] and office buildings [5] have shown that even in absence of a direct line-ofsight path between the transmitter and receiver, the temporal fading data show good fit to the Rician distribution.

Manuscript received November 3, 1993; revised April 4, 1994. This work was performed at TRLabs, Calgary, Alta., Canada during H. Hashemi's visit in the summer of 1993

H. Hashemi is with the Department of Electrical Engineering, Sharif University of Technology, Teheran, Iran.

M. McGuire is with the Department of Electrical Engineering, University of Victoria, Victoria, British Columbia, Canada.

T. Vlasschaert is with the Department of Computer Science, University of Calgary, Calgary, Alta., Canada.

D. Tholl is with Northern Telecom, Calgary, Alta., Canada.

IEEE Log Number 9403212
Temporal variation studies in office buildings where the environment is divided into separate rooms have shown that fading occurs in "bursts" lasting tens of seconds with a dynamic range of about $30 \mathrm{~dB}$ [5], [6]. For open office environments, however, fading was observed to be rather continuous with a dynamic range of $7 \mathrm{~dB}$ [5]. Width of the power spectrum of narrowband $\mathrm{CW}$ fading data has been estimated at $4 \mathrm{~Hz}$ for an office building [5]. A maximum value of $6.1 \mathrm{~Hz}$ has also been reported [7]. The "local" and "global" short time variations of the channel have been reported in [8]. This report also includes temporal variations of the rms delay spread and the received power.

Although the above works show some aspects of the temporal fading phenomenon, they do not report a number of important issues. For example, the type and degree of motion has not been a controlling factor in the measurements. Furthermore, other issues such as correlation properties, level crossing rates, and duration of fades statistics have not been addressed. In this paper, the results of extensive $\mathrm{CW}$ temporal fading measurements at 1100 $\mathrm{MHz}$ in a modern office environment under diversified sets of conditions are reported. In Section II of this paper the measurement plan and procedure are described. Section III presents the results of statistical analysis, followed by the concluding remarks of Section IV.

\section{Measurement Plan and Procedure}

Measurements were carried out at TRLabs premises in Calgary, Alta., Canada. This is a modern office environment with rooms, cubicles, hallways, and a large conference room. Four antenna separations of 5, 10, 20, and $30 \mathrm{~m}$ were considered. For each antenna separation four different base-portable antenna positions (configurations) were selected. In positioning base and portable antennas typical locations which included both line-of-sight (LOS) and non-light-of-sight (NLOS) topographies were chosen.

For each one of the 16 resulted cases (four antenna separations $\times$ four configurations per antenna separation), three types of motion were considered: motion around base antenna only, motion around portable antenna only, and motion around both antennas. The twelve recordings labeled (1B 0P), (2B 0P), (3B 0P), (4B 0P), (0B 1P), (0B 2P), (0B 3P), (0B 4P), (1B 1P), (2B 2P), (1B 2P), (1B $3 \mathrm{P})$ where $(\mathrm{iB} \mathrm{jP})$ represents motion of $i$ persons in the 
vicinity of the high ("base") antenna and $j$ persons in the vicinity of the low ("portable") antenna.

Each recording described above corresponds to $60 \mathrm{~s}$ of channel's temporal variations. During each $60 \mathrm{~s}$ care was taken to have continuous motion of the same nature. This means that if three people moved around the portable unit, they continued to do so throughout the whole $60 \mathrm{~s}$. Therefore, each 60 -s recording represents a sample from a stationary stochastic process. During each 1-min recording care was also taken to eliminate or minimize all other unplanned motion in the environment. To satisfy this latter requirement most measurements were carried out late at night.

The core of measurement setup was a HP $8510 \mathrm{C}$ network analyzer. One port of the network analyzer was connected to the base antenna and the other port to the portable antenna. A constant envelope tone at $1100 \mathrm{MHz}$ transmitted from the base antenna was received by the portable and passed to the network analyzer. The resulting signal was sampled at $50 \mathrm{~Hz}$. The data decimated to $50 \mathrm{~Hz}$ showed minimal loss of features compared to data from higher sampling frequencies.

Details of the measurement plan, measurement technique, equipment used, building structure, processing of data, etc. are reported in [9].

\section{Results of the Statistical Analysis}

\section{General Comments}

The 192 one-min recordings of the narrowband temporal fading data were analyzed extensively. Major results are briefly reviewed in this section.

Fig. 1 displays the envelop fading waveforms corresponding to different types and degrees of motion for antenna separation of $20 \mathrm{~m}$, configuration 3 . All figures are normalized to a mean of $0 \mathrm{dBm}$ and are plotted using the same scale. General features and properties of these figures are typical of most of the others.

Visual inspection of Fig. 1 shows that temporal variations are more noticeable for motion around the portable antenna, as compared to motion around the base antenna. Standard deviation and dynamic range of fluctuations are much smaller for the first four plots (1B OP, 2B OP, 3B OP, 4B OP) which involve motion around base only, as compared to the other eight plots which involve different degrees of motion around the portable antenna. A study of temporal fading waveforms has also shown that changes in signal level and dynamic range of fluctuations increased when the number of people around the portable antenna increased from 1 to 2 , and from 2 to 3 . However, changes were less noticeable for increase from 3 to 4 persons (a saturation phenomenon was observed for most cases).

\section{Amplitude Fading Distributions}

Empirical cumulative probability distribution function for each 1-min recording was obtained and tested against each one of five theoretical distributions normally en-
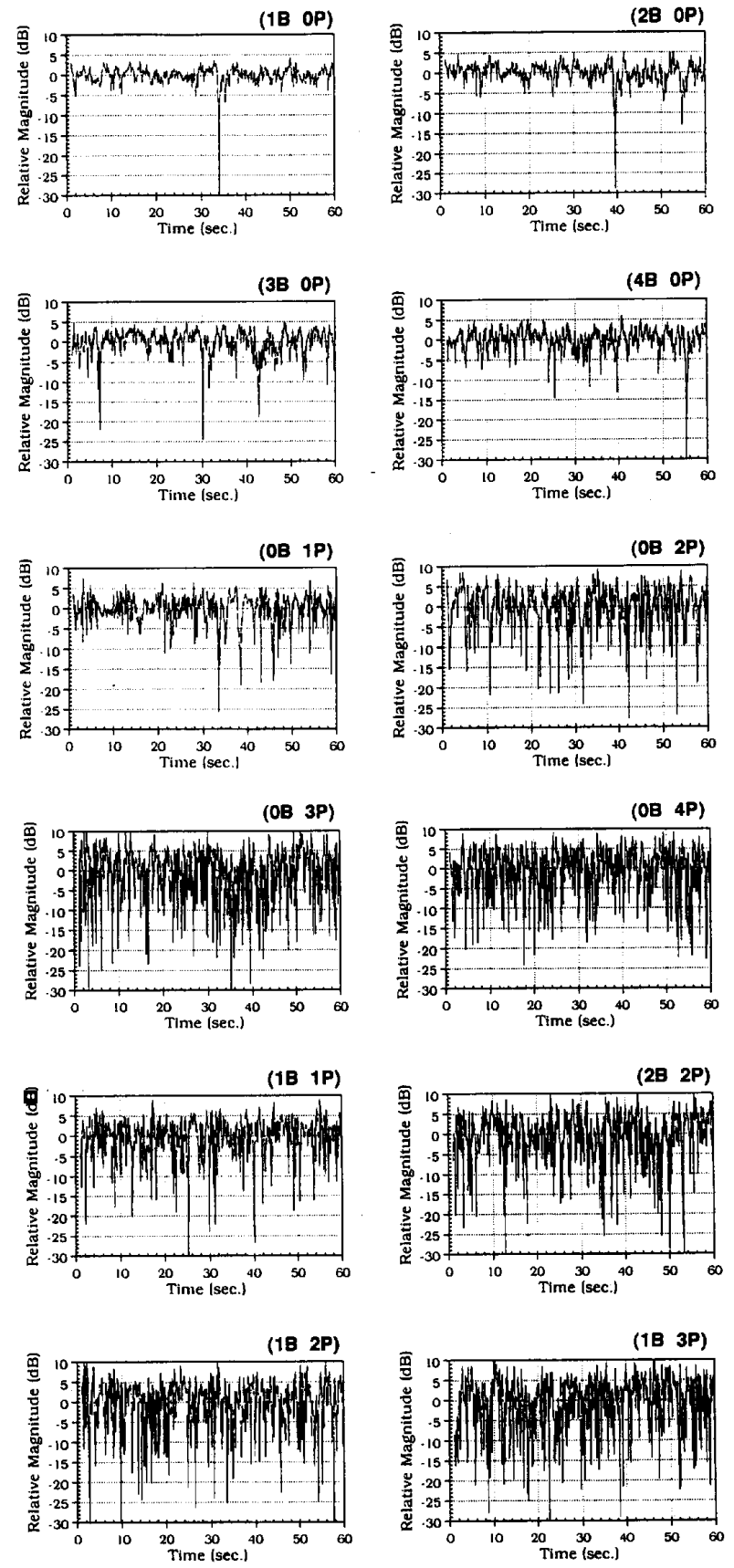

Fig. 1. Magnitude of narrowband temporal variation waveforms (in decibels relative to mean) for antenna separation of $20 \mathrm{~m}$, configuration 3 .

countered in describing multipath fading phenomenon (i.e., Rayleigh, Lognormal, Nakagami, Rice, and Weibull [1]).

The number of data points in each record $(60 \mathrm{~s} \times 50$ samples per second $=3000$ ) is large enough to provide statistically meaningful results. These samples are, of course, not independent. However, the correlation coefficient between the samples drops to less than 0.5 after 
$0.25 \mathrm{~s}$ for most records. Therefore, there are 240 " approximately" independent samples in each record. According to the Kolmogorov-Smirnov test [10], with this number of samples, the empirical distributions are within plus or minus $8 \%(=1.22 / \sqrt{n}$, with $n=240)$ of the true distributions, with a confidence level of $90 \%$.

To determine which distribution is the best for each record, the mean square error (MSE) test was employed. In this test the mean square error between the empirical and each one of the five theoretical cumulative probability distributions are calculated. The theoretical distribution with the smallest mean square error provides the best fit. Parameters of the theoretical distributions were estimated from the data using the moment method, in which the first and second moments of the theoretical distributions are equated with the corresponding empirical moments obtained from the data.

The best fit distribution for each type and degree of motion is shown in Table I. Examination of this table shows that the Weibull, Nakagami, Rice, Lognormal, and Rayleigh distributions show the best fit for $37 \%, 31.8 \%$, $22.3 \%, 7.8 \%$, and $1 \%$ of cases, respectively. Therefore, the Weibull and Nakagami distributions provide the best fit for most cases. Rayleigh fit is poor for almost all cases. Examination of Table I shows that: i) there is no specific trend between the best fit distribution and antenna separation; ii) good Weibull fit is more evident in LOS topographies (configurations 1 and 2 for the antenna separation of $5 \mathrm{~m}, 1$ for $10 \mathrm{~m}, 2$ for $20 \mathrm{~m}$, and 1 for $30 \mathrm{~m}$ ); iii) for motion around base only, the best fit distribution is often Nakagami, while for motion around portable the Weibull distribution fits better.

Variance of logarithmic data (in $\mathrm{dB}^{2}$ ) were calculated for each data record. The average values are tabulated in Table II, which also presents other statistical properties of the data. Each entry in the variance column of Table II is the average of 16 values corresponding to all cases having the same degree of motion (four antenna separations $x$ four configurations per antenna separation). It can be observed that when motion around the portable increases, on the average, the variance increases. Variances are also much higher for motion around portable, as compared to motion around base. The average value of fifth and tenth percentiles of the data are also included in Table II. It should be emphasized again that prior to analysis each data record was normalized to a mean value of 0 $\mathrm{dBm}$ (the $\mu$ column of Table II).

\section{Correlation Properties}

The correlation coefficient function $\rho(\Delta t)$ for amplitude fading at data points separated by $\Delta t$ was calculated. $\rho(\Delta t)$ is given by

$$
\rho(\Delta t)=\frac{E\{[a(t+\Delta t)-E(a(t+\Delta t))][a(t)-E(a(t))]\}}{\sqrt{\operatorname{Var}\{[a(t+\Delta t)]\} \operatorname{Var}\{[a(t)]\}}}
$$

where $a(t)$ is the amplitude along a time track of $1 \mathrm{~min}$, $E[\cdot]$ and $\operatorname{Var}[\cdot]$ denote the expected value and the vari-
TABLE I

Best Fit Amplitude Distributions. R: Rayleigh, Rı: Rice, N: NAKAGAMI, L: LOGNORMaL, W: WeIBULI

\begin{tabular}{|c|c|c|c|c|c|c|c|c|c|c|c|c|c|c|c|c|}
\hline \multirow{3}{*}{$\begin{array}{c}\text { Degree } \\
\text { of Motion }\end{array}$} & \multicolumn{16}{|c|}{ Antenna Separation } \\
\hline & \multicolumn{4}{|c|}{$5 \mathrm{~m}$} & \multicolumn{4}{|c|}{$10 \mathrm{~m}$} & \multicolumn{4}{|c|}{$20 \mathrm{~m}$} & \multicolumn{4}{|c|}{$30 \mathrm{~m}$} \\
\hline & 1 & 2 & 3 & 4 & 1 & 2 & 3 & 4 & 1 & 2 & 3 & 4 & 1 & 2 & 3 & 4 \\
\hline $1 B O P$ & w & $\mathrm{N}$ & $L$ & $\mathrm{~N}$ & w & $\mathbf{N}$ & $\mathrm{N}$ & $\mathrm{Ri}$ & $\mathbf{N}$ & $\mathrm{L}$ & $\mathrm{N}$ & $\mathrm{N}$ & $\mathrm{L}$ & $\mathrm{N}$ & $\mathrm{L}$ & $\mathrm{N}$ \\
\hline $2 B \quad O P$ & w & $\mathrm{N}$ & $\mathrm{Ri}$ & $\mathrm{N}$ & w & $\mathrm{N}$ & $\mathrm{N}$ & $\mathrm{Ri}$ & $\mathrm{N}$ & $\mathrm{N}$ & $\mathrm{L}$ & $\mathrm{N}$ & L & $N$ & $\mathrm{~N}$ & $\mathrm{~N}$ \\
\hline उB OP & w & $\mathrm{Ri}$ & $\mathrm{L}$ & $\mathrm{N}$ & w & $w$ & $\mathrm{~N}$ & $\mathrm{~N}$ & $\mathrm{~L}$ & $\mathrm{~N}$ & W & $\mathrm{N}$ & $\mathrm{N}$ & $\mathrm{N}$ & $\mathrm{N}$ & $\mathrm{N}$ \\
\hline $4 B \quad O P$ & w & $\mathrm{N}$ & $\mathrm{Ri}$ & $\mathrm{N}$ & w & $\mathrm{Ai}$ & $\mathrm{N}$ & Ri & $\mathrm{N}$ & $\mathrm{N}$ & $\mathrm{Ri}$ & $\mathrm{N}$ & $\mathrm{N}$ & $\mathrm{N}$ & $\mathrm{N}$ & $\mathrm{N}$ \\
\hline$O B \quad I P$ & w & L & $w$ & $\mathrm{~N}$ & $w$ & $w$ & N & $\mathrm{N}$ & $\mathrm{N}$ & $w$ & $\mathrm{Ri}$ & $w$ & L & $\mathrm{Ri}$ & $w$ & L \\
\hline$O B 2 P$ & w & $w$ & $w$ & $\mathrm{~L}$ & $\mathrm{~N}$ & qi & $\mathrm{N}$ & $w$ & $\mathrm{~N}$ & w & 9i & $\mathrm{Ri}$ & $w$ & w & w & $\mathrm{Ri}$ \\
\hline$O B \quad 3 P$ & $\mathrm{Ri}$ & $w$ & $\mathrm{~L}$ & $\mathbf{R i}$ & w & $\mathrm{Ai}$ & $\mathrm{N}$ & $\mathrm{Ri}$ & Ri & w & w & $\mathrm{N}$ & $w$ & w & $\mathrm{N}$ & $\mathrm{N}$ \\
\hline$O B A P$ & $\mathrm{Ri}$ & $w$ & $w$ & $\mathrm{Ri}$ & $\mathrm{Ri}$ & $\mathrm{Ri}$ & $\mathrm{N}$ & $w$ & $\mathrm{Ri}$ & w & $w$ & $w$ & R & w & $w$ & $\mathrm{R}$ \\
\hline 1B IP & $w$ & $w$ & $w$ & $N$ & $w$ & $\mathrm{Ri}$ & $w$ & $w$ & $\mathrm{~N}$ & w & $\mathrm{Ri}$ & Ai & $\mathrm{Ri}$ & $\mathrm{Ri}$ & $w$ & $N$ \\
\hline $2 \mathrm{~B} 2 \mathrm{P}$ & w & $w$ & $w$ & $\mathrm{Ri}$ & $w$ & $\mathrm{Ri}$ & $\mathrm{N}$ & $w$ & $\mathrm{~N}$ & W & w & $\mathrm{N}$ & w & w & w & $\mathrm{Ri}$ \\
\hline $1 \mathrm{~B} 2 \mathrm{P}$ & Pi & $w$ & L & $\mathrm{Ri}$ & Ri & $\mathrm{Ri}$ & Ai & w & $\mathrm{N}$ & W & $\mathrm{N}$ & $\mathrm{Ai}$ & $w$ & $w$ & $w$ & $\mathrm{Ri}$ \\
\hline $1 \mathrm{BP}$ & $w$ & $\mathrm{Bi}$ & $w$ & $\mathrm{Fi}$ & $\mathrm{N}$ & $\mathrm{Ri}$ & $w$ & $\mathrm{~N}$ & $\mathrm{Ri}$ & W & w & $w$ & $w$ & $L$ & $W$ & $\mathrm{~N}$ \\
\hline
\end{tabular}

TABLE II

Statistical Parameters of the Temporal Fading Data. Each Entry is the Average of 16 Values ( 4 antenna Separations $\times 4$ Configurations)

\begin{tabular}{|c|c|c|c|c|c|c|c|c|c|c|c|c|}
\hline \multirow{2}{*}{$\begin{array}{c}\text { Degree } \\
\text { of Motion }\end{array}$} & \multicolumn{2}{|c|}{ Moments } & \multicolumn{2}{|c|}{ Percentiles } & \multicolumn{2}{|c|}{ Corr. Coeff. } & \multicolumn{2}{|c|}{ LCR } & \multicolumn{2}{|c|}{ Fade Dur. $(\%)$} & \multicolumn{2}{|c|}{ Spect. Width } \\
\hline & $\mu$ & $\sigma^{2}$ & 5th & 10th & $0.2 \mathrm{~s}$ & $0.5 \mathrm{~s}$ & $-5 \mathrm{~dB}$ & $-10 \mathrm{~dB}$ & $-5 \mathrm{~dB}$ & $-10 \mathrm{~dB}$ & $-3 \mathrm{~dB}$ & $-20 \mathrm{~dB}$ \\
\hline & 0 & 1.4 & -1.7 & -1.2 & 0.52 & 0.22 & 1.6 & 0.1 & 0.3 & 0.0 & 0.28 & 1.41 \\
\hline $2 B O P$ & 0 & 2.3 & -2.5 & -1.8 & 0.53 & 0.25 & 3.6 & 0.2 & 0.6 & 0.0 & & 3.09 \\
\hline $380 F$ & 0 & 3.8 & -3.0 & -2.2 & 0.50 & 0.23 & 9.9 & 1.6 & 1.7 & 0.3 & 0.33 & 3.95 \\
\hline 48 OP & 0 & 4.4 & -3.3 & -2.3 & 0.49 & 0.21 & 12.0 & 2.7 & 2.0 & 0.3 & 0.35 & 4.94 \\
\hline & 0 & 8.7 & -4.7 & -3.1 & 0.60 & 0.34 & 18.7 & 6.7 & 4.9 & 1.2 & 0.34 & 5.41 \\
\hline $092 P$ & 0 & 16.4 & -6.8 & .4 .6 & 0.56 & 0.31 & 35.6 & 15.1 & 9.4 & 2.6 & 0.28 & 1.41 \\
\hline $083 P$ & 0 & 18.5 & .7 .2 & -5.0 & 0.51 & 0.24 & 51.8 & 24.3 & 10.6 & 2.8 & 0.32 & 5.14 \\
\hline 0848 & 0 & 21.6 & -8.2 & -5.7 & 0.47 & 0.21 & 58.9 & 31.6 & 12.5 & 3.7 & 0.40 & 6.34 \\
\hline & 0 & 12.8 & -5.9 & -4.1 & 0.58 & 0.29 & 27.3 & 12.8 & 7.9 & 1.9 & 0.45 & 7.32 \\
\hline $2 \mathrm{~B} \quad 2 \mathrm{P}$ & 0 & 17.3 & -6.8 & -4.6 & 0.50 & 0.21 & 42.3 & 19.0 & 9.7 & 2.6 & 0.49 & 7.83 \\
\hline IB $2 P$ & 0 & 16. & -6.7 & -4.5 & 0.51 & 0.23 & 44.6 & 18.9 & 9.7 & 2.4 & 0.28 & 1.39 \\
\hline $1 \mathrm{~B} 3 \mathrm{P}$ & 0 & 20.5 & -7.7 & -5.2 & 0.48 & 0.21 & 55.5 & 27.9 & 11.6 & 3.2 & 0.36 & 6.24 \\
\hline
\end{tabular}

ance of $[\cdot]$, respectively, $t$ is time (ranging from 0 to 60 $\mathrm{s})$, and $\Delta t$ is temporal separations in multiples of one sampling time (i.e., $0.02 \mathrm{~s}$ ).

Average magnitude of $\rho(\Delta t)$ versus $\Delta t$ for antenna separation of $5 \mathrm{~m}$, configuration 1 is shown in Fig. 2. Average magnitude of $\rho(\Delta t)$ for $\Delta t=0.2$ and $0.5 \mathrm{sec}$. are tabulated in Table II. For most cases $|\rho(\Delta t)|$ drops to less than 0.2 after $1 \mathrm{~s}$

\section{Level Crossing Rates}

Level crossing rate (LCR) is the number of times that the signal crosses a specified threshold with a positive slope. LCR was calculated for each record and for threshold levels ranging from $-20 \mathrm{~dB}$ to $+10 \mathrm{~dB}$. The results for antenna separation of $20 \mathrm{~m}$, configuration 4 are reproduced in Fig. 3. Average LCR values for each degree of motion and for threshold levels of $-5 \mathrm{~dB}$ and $-10 \mathrm{~dB}$ are tabulated in Table II. LCR plots for motion around the portable are, in general, more scattered, as compared to motion around the base. This means that a given threshold level is crossed more frequently for motion around the portable. Also, for most cases as the number of people in motion around the portable unit increases LCR increases. This is consistent with the average LCR values of Table II and with the results obtained by visual inspection of Fig. 1. 


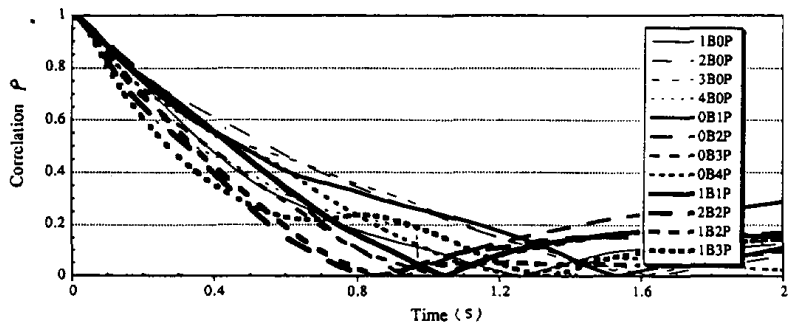

Fig. 2. Average of magnitude of correlation coefficients for antenna separation of $5 \mathrm{~m}$, configuration 1 .

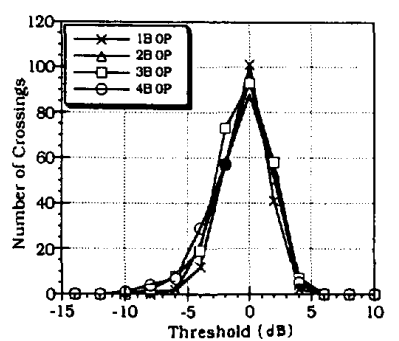

(a)

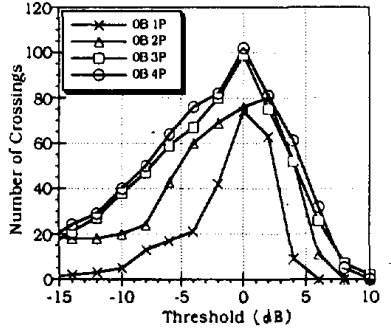

(b)
Fig. 3. Level crossing rates (LCR) for antenna separation of $20 \mathrm{~m}$, configuration 4. (a) Motion around base, (b) motion around portable.

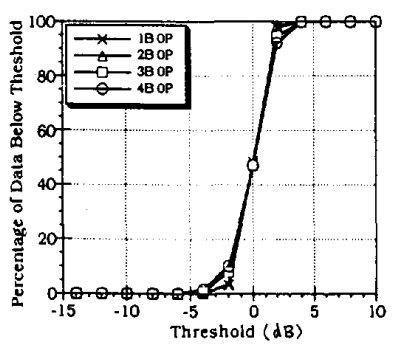

(a)

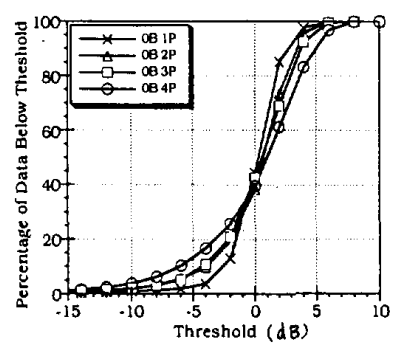

(b)
Fig. 4. Percentages of data below a given threshold versus threshold for antenna separation of $5 \mathrm{~m}$, configuration 4. (a) Motion around base, (b) motion around portable.

\section{Duration of Fades Statistics}

Performance of indoor communication systems is sensitive not only to the number of times that a low threshold is crossed, but also to the duration of time that the signal stays below the given threshold. The percentage of time that the signal remains below a threshold was calculated for each record and for different threshold levels. The results for antenna separation of $5 \mathrm{~m}$, configuration 4 are shown in Fig. 4. Average values for all data representing the same type and degree of motion (threshold levels of $-5 \mathrm{~dB}$ and $-10 \mathrm{~dB}$ ) are included in Table II.

The conclusion drawn for the LCR also applies to these "fade durations"': duration of fades is higher for motion around the portable, as compared to motion around the base. For most cases fade durations (for negative threshold levels) increase as the number of people in motion around the portable increases.
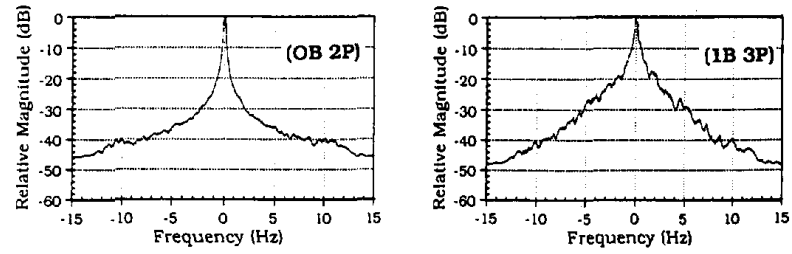

Fig. 5. Magnitude spectrum of the narrowband fading waveforms (in decibels relative to maximum) for antenna separation of $30 \mathrm{~m}$, configuration 2.

\section{Frequency Domain Analysis}

The temporal variation data were transferred to frequency domain using classical Fourier analysis. Typical smoothed magnitude responses are shown in Fig. 5. These plots are normalized to a maximum of $0 \mathrm{~dB}$.

The spectrum width for levels of $3,5,10$, and $20 \mathrm{~dB}$ below the maximum were calculated for each data record. The average spectrum width corresponding to $-3 \mathrm{~dB}$ and $-20 \mathrm{~dB}$ points are tabulated in Table II. The overall mean spectrum widths (average of all 192 one-min recordings) for $-3,-5,-10$, and $-20 \mathrm{~dB}$ points are $0.35,0.48$, 1.06 , and $4.54 \mathrm{~Hz}$, respectively.

\section{ConClusion}

Temporal variations of the indoor radio propagation channel have been studied extensively using an elaborate measurement plan which resulted in establishing a large data base of $11520 \mathrm{~s}$ of narrowband $\mathrm{CW}$ envelope fading data. The analysis included both qualitative evaluation of the variations, and quantitative analysis consisting of the amplitude fading distributions, correlation properties, level crossing rates, fade durations, and frequency domain properties.

The results reported in this paper can be used in the design of wireless indoor radio communication systems. They are particularly useful for high data rate wireless computer communications applications where both terminals are stationary but motion of people results in multipath distortions in the received signal. With proper interpretations and further analysis the results can also lead to a model that simulates temporal variations on the channel under diversified sets of conditions.

A particularly challenging research area which is being pursued now is to "superimpose" the CW temporal variation information on wideband spatial-variation models [1]-[3]. Such an undertaking, however, requires extensive mathematical and statistical modeling efforts.

\section{ACKNOWLEDGMENT}

H. Hashemi would like to thank J. McRory for his support and encouragement throughout this project.

\section{REFERENCES}

[1] H. Hashemi, "The indoor radio propagation channel," Proc. IEEE, vol. 81, no. 7, pp. 943-968, July 1993 .

[2] _ - "Impulse response modeling of indoor radio propagation chan- 
nels,"' IEEE J. Select. Areas Commun., vol. 11, no. 7, pp. 967-978, Sept. 1993.

[3] H. Hashemi and D. Tholl, "Statistical modeling and simulation of the RMS delay spread of indoor radio propagation channels," IEEE Trans. Veh. Technol., vol. 43, no. 1, pp. 110-120. Feb. 1994.

[4] T. S. Rappaport and C. D. McGillem, "UHF fading in factories," IEEE J. Select. Areas Commun., vol. 7, pp. 40-48, Jan. 1989.

[5] R. J. C. Bultitude, "Measurement, characterization and modeling of indoor $800 / 900 \mathrm{MHz}$ radio channels for digital communications," IEEE Commun. Mag., vol. 25, no. 6, pp. 5-12, June 1987.

[6] R. J. C. Bultitude, S. A. Mahmoud, and W. A. Sullivan, "A comparison of indoor radio propagation characteristics at $910 \mathrm{MHz}$ and $1.75 \mathrm{GHz}$," IEEE J. Select. Areas Comm., vol. 7, pp. 20-30, Jan. 1989.

[7] S. J. Howard and K. Pahlavan, " Doppler spread measurements of the indoor radio channels," Electron. Lett., vol. 26, pp. 107-109, Jan. 1990

[8] R. Ganesh and K. Pahlavan, "Statistics of short time variations of indoor radio propagation," in Proc. Internat. Conf. Commun. ICC'91, Denver, CO, June 23-26, 1991.

[9] H. Hashemi, M. McGuire, T. Vlasschaert, and D. Tholl, "A study of temporal variations of the indoor radio propagation channel," TRLabs Internal Rep. Sept. 1993.

[10] W. J. Dixon and F. J. Massey, Introduction to Statistical Analysis. New York: McGraw Hill, 1969, 3rd ed

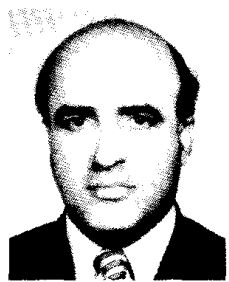

Homayoun Hashemi (M'89-SM'93) was born in Teheran, Iran. He received the B.S.E.E. degree from the University of Texas at Austin, in 1972, and the M.S. and Ph.D. degrees in electrical en gineering and computer sciences from the University of California at Berkeley, in 1974 and 1977 , respectively. He also received the Master's degree in statistics from the University of California at Berkeley.

In the summer of 1977 , he served as a Consult ing Engineer at Stanford Research Institute, Menlo Park, California. From 1977 to 1979 , he was a member of the Technical Staff at Bell Telephone Laboratories, Holmdel, NJ, where he was involved in system design for high-capacity mobile telephone systems. Since 1979 he has been a faculty member in the Electrical Engineering Department of Sharif University of Technology, where he is currently Associate Professor. He was on sabbatical leave at NovAtel Communications Ltd. in Calgary, Alberta, Canada in the 1990-91 academic year, where he worked on various projects related to mobile and personal communications. He spen the summers of 1992 and 1994 at the Electrical Engineering Department of the University of Ottawa, where he was involved in propagation modeling at infrared frequencies for indoor wireless communications. He spent the summer of 1993 at TRLabs, Calgary, Alberta, Canada, supervising several projects related to indoor radio propagation modeling and PCS system design. He has worked on different aspects of wireless communications, including general system architecture, channel assignment, propagation modeling, performance analysis, and capacity evaluations for digital cellular, microcellular, and indoor wireless communication systems. His channel simulation package SURP has been used internationally in the design of digital cellular radio communication systems. He has also served as a consultant to the Iranian Telecommunications Research Center and the Telecommunications Company of Iran.

M. McGuire, photograph and biography not available at the time of publication.

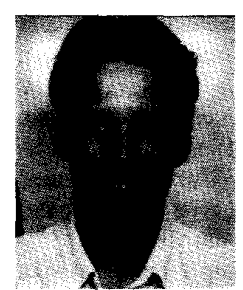

Thomas Vlasschaert was born in Sarnia, Ont., Canada in 1972. He is currently completing the B.Sc. degree in computer science at the University of Calgary and expects to receive the degree in April 1995. His contributions to this project were completed at TRLabs Wireless Research facility in Calgary as cooperative education credit towards his degree.

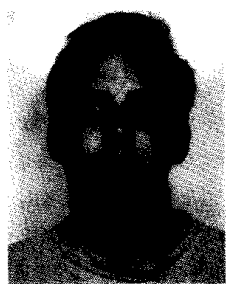

David Tholl (SM'93) was born in Regina, Saskatchewan, Canada. He received the B.Sc. degree with Distinction in electrical engineering from the Unversity of Alberta in 1989 and is currently completing the M.Sc. degree in electrical and computer engineering on a part-time basis at the University of Calgary.

From 1989 to 1991, he worked as an RF Engineer in the antennas and propagation group of NovAtel Communications Ltd. where he was involved in the measurement of fading channels and diversity experiments. He had a key role in establishing a large database of indoor radio propagation channel measurements. He took active participation in a project comparing the performance of various channel measurement techniques. During his Master's research he was involved with and funded by the wireless thrust of Telecommunications Research Laboratories, TRLabs, where he performed statistical modeling of the indoor radio propagation channel. He joined the PCS product development group of Northern Telecom, Calgary, Alta., in 1993 where he is engaged in the development of CT-2+ equipment. 